\title{
Influence of Phase Behavior and Miscibility on Mechanical, Thermal and Micro-Structure of Soluble Starch-Gelatin Thermoplastic Biodegradable Blend Films
}

\author{
Emad Ali Soliman ${ }^{1,2 *}$, Masakazu Furuta² \\ ${ }^{1}$ Department of Polymeric Materials, Institute of Advanced Technology and New Materials, City of Scientific \\ Research and Technology Applications, Alexandria, Egypt \\ ${ }^{2}$ Department of Quantum and Radiation Engineering, Graduate School of Engineering, Osaka Prefecture \\ University, 1-1 Gakuen Cho, Sakai, Osaka, Japan \\ Email: ${ }^{*}$ emadsoliman@mucsat.sci.eg
}

Received 16 March 2014; revised 20 April 2014; accepted 30 April 2014

Copyright $@ 2014$ by authors and Scientific Research Publishing Inc.

This work is licensed under the Creative Commons Attribution International License (CC BY). http://creativecommons.org/licenses/by/4.0/

(c) (9) 0pen Access

\section{Abstract}

Polymer blends of cold water soluble starches (amylose or amylopectin soluble starch) with gelatin were prepared using solvent casting method. The solid state miscibility and polymer-polymer interactions between the constituent polymers were studied by fourier transforms infrared spectroscopy (FTIR), X-ray diffraction (XRD), differential scanning calorirmetry (DSC), light optical microscopy (OP) and scanning electron microscopy (SEM), whereas the thermal stability of the blends was studied by thermogravimetric analysis (TGA). Furthermore, tensile and water vapor barrier properties of the blends were assessed. The obtained results exhibited that gelatin was more miscible with amylose soluble starch than with amylopectin soluble starch. Moreover, enhancing mechanical and water barrier properties of amylose soluble starch/gelatin blends were more pronounced than those of amylopectin soluble starch/gelatin blends. Generally, tensile strength (TS) and Elongation percentage (E) of the blend films were found to be gradually increased with increasing the proportion of gelatin. Nevertheless, increasing starch proportion was in favor of decreasing water vapor permeability (WVP). At equal proportions of starch and gelatin (1:1), TS was raised up to 8.69 MPa for amylose soluble starch/gelatin blend films while it raised up to 4.96 MPa for amylopectin soluble starch/gelatin blend films, and so on E was increased to its maximum by $\sim \mathbf{1 7 9 . 6 \%}$ for soluble amylose starch/gelatin blends while it was increased to $\sim 114.5 \%$ for amylopectin soluble starch/gelatin blends. On the other hand, WVP was significantly decreased to be 6.46 and $12.09 \mathrm{~g} \cdot \mathrm{mm} / \mathrm{m}^{2} \cdot d a y \cdot \mathrm{kPa}$ for blends of amylose and amylopectin soluble starches,

"Corresponding author.

How to cite this paper: Soliman, E.A. and Furuta, M. (2014) Influence of Phase Behavior and Miscibility on Mechanical, Thermal and Micro-Structure of Soluble Starch-Gelatin Thermoplastic Biodegradable Blend Films. Food and Nutrition Sciences, 5, 1040-1055. http://dx.doi.org/10.4236/fns.2014.511115 
respectively.

Keywords

Soluble Amylose Starch, Soluble Amylopectin Starch, Gelatin, Biodegradable Blend Films

\section{Introduction}

At ends of the last century, development of novel materials from the renewable resources has been receiving growing attention [1]. Where, natural polymers have replaced conventional synthetic polymers originating from petroleum resources in various applications because most of the latter are regarded as non-biodegradable which its widening use has caused serious environmental problems especially when they are used in production of disposable and non-durable items with short period of use in addition to the petroleum is considered exhausted resource [2]. Among these natural polymers, starch with its major components, amylose and amylopectin are currently being used in the production of biodegradable packaging materials. The development of starch-based biodegradable plastic materials is becoming an increasingly attractive alternative to petroleum-based products because of its renewability and low cost. However, wide application of starch-based films has limited by their moisture sensitivity and brittleness [3]. This constraint has led to the development of the improved properties of starch-based materials by modifying its starch properties and/or incorporating other polymer materials.

Blending of polymers was a matter of active interest mainly due to being one of the simplest ways to develop new materials with a variety of designed physical and chemical properties at the lowest cost. The gain in newer properties depends on the degree of compatibility or miscibility of the polymers at a molecular level. Thus, the chemical structure of the constituent polymers plays a determining role in the polymer-polymer interactions. Consequently, polymer-polymer interactions determine the phase behavior of constituent polymers either miscibility, phase separation or various levels of mixing in between the extremes (e.g., partial miscibility). Investigating the phase behavior is of interest in the prediction of the full set of desired specific functional properties of polymer blends [3] [4].

Miscibility may arise from specific interactions, such as hydrogen bonding, dipole-dipole forces and charge transfer complexes for polymer mixtures or segment-segment repulsion inside the blends which lead to form interacting single-phase polymer blends [4]. These interactions give rise to negative heats of mixture favoring the mixing process [5]. However, a majority of known polymer blends are immiscible due to entropic reasons where, a very small entropy gain in mixing long polymer chains and because of the usually encountered positive heat of mixing, leading to formation of separated phases.

Since, the functional groups on starch i.e., hydroxyl groups could interact with carboxyl amino, and/or hydroxyl groups on gelatin chains via forming attractive hydrogen bonding interactions, blending starch with different proteins (casein, gelatin, and albumin) to decrease the water vapor permeability of the films and to increase their tensile strength have been studied. However, casein-based film showed a lower water-vapor transmission rate, water gain at different relative humidity conditions, and higher tensile strength compared to its counterparts containing gelatin and albumin [6].

The native starches do not possess the physicochemical attributes necessary to meet the very diverse range of functional properties required by various applications. Therefore, in most cases, it is necessary to chemically or physically modify the structure of native starch granules present in the crystalline region, or decrease the size of crystalline regions to increase reaction activity of starch to improve their cold water solubility that will inevitably have an impact on its functional properties including: viscosity, clarity, water retention, reactivity, freezethaw stability and adhesive properties [7]. Therefore, the objective of this study was to develop biodegradable polymer blend films from two different types of starches, i.e., amylopectin or amylose soluble starch with gelatin using solvent evaporation casting technique. The solid state miscibility was studied by analyzing Fourier transform infrared spectroscopy (FT-IR), differential scanning calorimetry (DSC), X-ray diffraction (XRD), optical microscopy (OP) and scanning electron microscopy (SEM) of blend films. The thermal degradation behaviors of the blends were studied by thermogravimetric analysis. The studies relating to determination of mechanical properties and water vapor barrier properties of the films were carried out. 


\section{Materials and Methods}

\subsection{Materials}

Modified water-soluble potato starch (70\% - 75\% amylopectin) and modified water-soluble corn starch (70\% amylose) were obtained from (Panreac, a.r., Barcelona, Spain). Gelatin was purchased from Sigma-Aldrich; Chemie GmbH. An analytical grade with $99.5 \%$ purity of glycerol, as a plasticizer, was obtained from Chem. Supply Pty. Ltd., SA, Australia.

\subsection{Preparation of Blends}

Aqueous film-forming solutions with selected amounts of soluble starch, gelatin, and glycerol were prepared in accordance with the experimental design (Table 1). Blend films were prepared as previously proposed by Soliman et al. [8]. The solutions were heated $90^{\circ} \mathrm{C} \pm 2^{\circ} \mathrm{C}$ with mixing using mechanical stirrer on a hot plate for 30 min. Each solution was casted onto polystyrene casting plates (Figure 1) and dried at $30^{\circ} \mathrm{C} \pm 2{ }^{\circ} \mathrm{C}$ in a vacuum oven. Finally, the detached blend films from casting plates were conditioned at $60 \% \pm 2 \% \mathrm{RH}$ and $27^{\circ} \mathrm{C} \pm 2{ }^{\circ} \mathrm{C}$ in controlled environment chamber.

\subsection{ATR-FTIR Spectroscopic Analysis}

ATR-FTIR spectroscopic analysis was carried out using Shimadzu AIM8400 FTIR spectrometer equipped with ATR-8400M objective (Shimadzu Co, Ltd., Tokyo, Japan). This system adopted a germanium prism tip. The spectra were recorded over a range of $500-4000 \mathrm{~cm}^{-1}$ with a resolution of $2 \mathrm{~cm}^{-1}$ and averaged 32 scans.

\subsection{Differential Scanning Calorimetric (DSC) Analysis}

Glass transition temperature $\left(T_{g}\right)$, enthalpy $(\Delta H)$ and mass fraction crystallinities for the obtained blends were measured using Shimadzu DSC-60A calorimeter (Shimadzu Co. Ltd., Kyoto, Japan) equipped with a data processor. DSC was first calibrated with indium, and purged with nitrogen gas atmosphere. The samples (10 mg) were weighed in an aluminum pan, allowed to equilibrate to $25^{\circ} \mathrm{C}$, and scanned from $25^{\circ} \mathrm{C}$ to $350^{\circ} \mathrm{C}$ at a constant heating rate of $5^{\circ} \mathrm{C} / \mathrm{min}$. Crystallization degree for melt and for each of the polymer blend constituents was calculated by the following equation:

Table 1. Content of film-forming components.

\begin{tabular}{cccc}
\hline Trial & Starch $(\mathrm{w} / \mathrm{w} \%)$ & Gelatin $(\mathrm{w} / \mathrm{w} \%)$ & Glycerol $(\mathrm{w} / \mathrm{w} \%)$ \\
\hline 1 & 0 & 7.0 & 0.2 \\
2 & 3.5 & 3.5 & 0.2 \\
3 & 4.2 & 2.8 & 0.2 \\
4 & 4.9 & 2.1 & 0.2 \\
5 & 5.6 & 1.4 & 0.2 \\
6 & 6.3 & 0.7 & 0.2 \\
7 & 7.0 & 0 & 0.2 \\
\hline
\end{tabular}

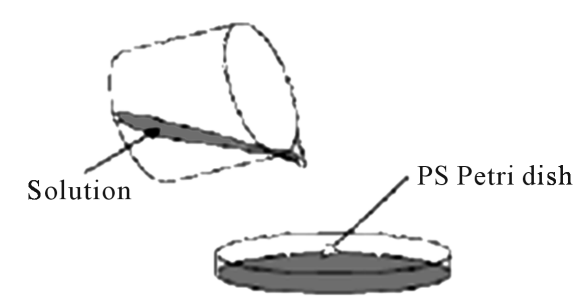

Figure 1. Casting technique. 


$$
X_{c}=\frac{\Delta H_{c}}{W_{c}} . \Delta H_{m}^{\circ} \times 100
$$

where, $\Delta H_{c}$ denotes the melt crystallization enthalpy of each one of the blend constituents, $w_{c}$ refers to the weight fraction of this constituent, and $\Delta H_{m}^{\circ}$ is the enthalpy of fusion of $100 \%$ crystalline component which based on the proven data reported in the literature $\left(\Delta H_{m}^{\circ}=211.4 \mathrm{~J} / \mathrm{g}\right.$ for chitosan, $69.8 \mathrm{~J} / \mathrm{g}$ for PVA, $62.05 \mathrm{~J} / \mathrm{g}$ for gelatin and $128.8 \mathrm{~J} / \mathrm{g}$ for potato starch [9]). The same equation in which $\Delta H_{c}$ is replaced with cold crystallization enthalpy was applied to estimate the degree of crystallinity for solid state.

\subsection{X-Ray Diffraction (XRD)}

X-ray diffraction analysis for plain and blend films were obtained using a Shimadzu XRD-7000 diffractometer (Shimadzu Co. Ltd., Japan) (30 kV, $30 \mathrm{~mA}$ ) with $\mathrm{Cu} \mathrm{K \alpha}$, Ni-filtered radiation and equipped with the computer application software (DP-D1, Shimadzu Co. Ltd.). The film specimen was layered on a sample holder and scanned from $4^{\circ}-60^{\circ}$ at rate of $2^{\circ} / \mathrm{min}$. The crystallinity is calculated by separating intensities due to amorphous and crystalline phases on diffraction phase. Since computer aided curve resolving technique is used to separate crystalline and amorphous phases of diffractogram. Total area on X-ray diffraction pattern can be divided into crystalline $(A c)$ and amorphous components $(A a)$ (Figure 2). Crystallinity percentage $(X c \%)$ can be assigned by proportion of crystalline area to total area as indicated in the following equation;

$$
X c \%=\frac{A c}{A c+A a} \times 100
$$

where;

$A c=$ Area of crystalline phase

$A a=$ Area of amorphous phase

$X_{C}=$ Degree of crystallinity

\subsection{Thermogravimetric Analysis (TGA)}

The thermal analysis was performed by using Shimadzu TGA-50 Analyzer (Shimadzu Co. Ltd., Kyoto, Japan). Specimens $(10 \mathrm{mg})$ were thermally scanned from $25^{\circ} \mathrm{C}$ to $900^{\circ} \mathrm{C}$ in open corundum crucibles under nitrogen with flow rate of $\sim 200 \mathrm{ml} / \mathrm{min}$ at scanning rate $10^{\circ} \mathrm{C} / \mathrm{min}$.

\subsection{Mechanical Properties Testing}

Tensile strength (TS) and elongation (E) at break were measured with a Shidmadzu AG-I 5 kN universal testing machine (Shimadzu Co. Ltd., Kyoto, Japan) according to ASTM D882-00 Standard Method [11]. Ten film specimens of $2.54 \mathrm{~cm} \times 12 \mathrm{~cm}$, were cut from ten tested films. Initial grip separation and crosshead speed were set at $50 \mathrm{~mm}$ and $50 \mathrm{~mm} / \mathrm{min}$, respectively. Tensile strength was calculated by dividing the maximum force by initial specimen cross-sectional area, and percent elongation at break was calculated as follows:

$$
E=\left(d_{\text {after }}-d_{\text {before }}\right) / d_{\text {before }} \times 100
$$

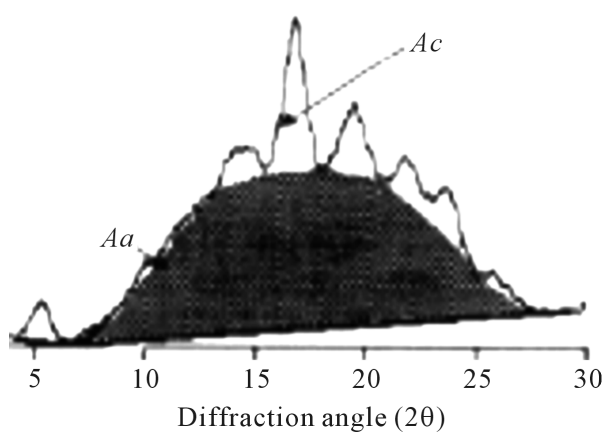

Figure 2. Schematic diagram presenting the relative degree of crystallinity. 
where, $d$ was the distance between grips holding the specimen before or after the break of the specimen.

\subsection{Water Vapor Permeability (WVP) Measurement}

Water vapor transmission rate (WVTR) of blend films was determined by using Permatran W600 (Macon Inc., Minneapolis, MN). This measurement was done according to ASTM E 96-00 standard method [12]. It is sixstation computer-monitored system. Blend film specimens were tested at $23^{\circ} \mathrm{C}, 60 \% \mathrm{RH}$ gradient over $0 \% \mathrm{RH}$ on one side of the film with air flow of $10 \mathrm{~mL} / \mathrm{min}$. Permatran was calibrated with Mylar film $0.13 \mathrm{~mm}$ (5 mil) thick. Stainless-steel masks were used to adjust the transmission area to $20 \mathrm{~cm}^{2}$. WVP was calculated and expressed as $\mathrm{g} \cdot \mathrm{mm} / \mathrm{m}^{2} \cdot$ day $\cdot \mathrm{kPa}$. Four replicates were tested for each tested film.

\subsection{Light Optical Microscopy}

Morphology of the prepared films was examined by using an Olympus BH2-UMA microscope attached with Canon photometric high resolution digital camera. The specimen was first stained with iodine solution before examination. Magnification power used in examination was 500 or $1000 \times$.

\subsection{Scanning Electron Microscopy (SEM)}

The microstructure of the plain and blend films was characterized by JEOL JSM-6360 LA scanning electron microscope (JEOL Ltd., Rigaku, Tokyo, Japan). Examination was performed at an acceleration voltage of $15 \mathrm{kV}$ with magnification power of 1000 - 3000×. Film samples were first coated with gold using JFC-1100E sputtering coater.

\subsection{Statistical Analysis}

A completely randomized experimental design was used to study the component ratios on the properties of biodegradable starch/gelatin blend films. Analysis of variance (ANOVA) was used to determine the effect of type of soluble starches and their ratios. Multiple comparisons were performed using Duncan's Multiple Range Test (DMRT).

\section{Results and Discussion}

\subsection{Compositional Properties}

FT-IR spectra of plain films of soluble starch (ASS and ApSS) and gelatin (G) are shown in Figure 3(A)(a), Figure 3(B)(a) and Figure 3(A), Figure 3(B)(c) respectively. It can be concluded that amylose and amylopectin cannot be distinguished by IR spectroscopy. In FT-IR spectrum of both types of soluble starches, a broad absorption band appeared at $\sim 3300 \mathrm{~cm}^{-1}$, assigned to -OH stretching frequency, and a band at $2940 \mathrm{~cm}^{-1}$ attributable to C-H stretching vibrations. The presence of a strong absorption band at $1638 \mathrm{~cm}^{-1}$ confirms the presence of water. The bands around 1414 and $1338 \mathrm{~cm}^{-1}$ are assigned to $-\mathrm{CH}_{2}$ bending in plane and C-OH bending vibrations, respectively. The band at $1148 \mathrm{~cm}^{-1}$ is due to C-O-C anti-symmetric bridge stretching. In despite of occurrence some differences in the intensity of certain adsorption bands and shift, these IR spectra cannot distinguish amylose and amylopectin soluble starches [13]. FTIR spectrum of gelatin films exhibited absorbance bands at 1631 and $1538 \mathrm{~cm}^{-1}$ assigned to $\mathrm{C}=\mathrm{O}$ stretching (amide I), and angular deformation of N-H (amide II), respectively. In addition to adsorption bans appeared at $1448 \mathrm{~cm}^{-1}$ due to $\mathrm{C}-\mathrm{H}$ deformation. The absorption band at $1236 \mathrm{~cm}^{-1}$ is attributed to C-N stretching and the vibrational band of $\mathrm{N}-\mathrm{H}$ (amide III). The broad band from $3600-3100 \mathrm{~cm}^{-1}$ was the O-H and or N-H stretching [14]. The band at $2930 \mathrm{~cm}^{-1}$ was C-H stretching [15].

These spectra of blend films (AAS/G and ApSS/G) have characteristic traits of both starch and gelatin films, both starch-gelatin blend films exhibited the same characteristic FT-IR spectra (Figure 3(A), Figure 3(B)(b)). However, it was observed that ASS confers its characteristic traits on the spectrum of the resultant blend than what did amylopectin. This can be attributed to specific interactions like dipole-dipole forces, hydrogen bonding and charge transfer complexes that are responsible for good miscibility at molecular level between amylose soluble starch and gelatin in the blend film matrix. On the contrary, amylopectin soluble starch could not be the dominant in its blends with gelatin. This can be explained on the basis of the partial miscibility because of polymer-polymer interactions normally lose their capacity of to return to their native forms [16]. 


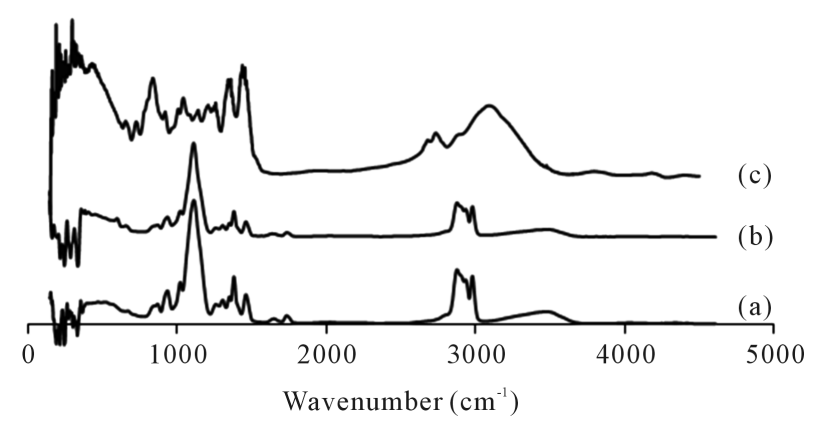

(A)

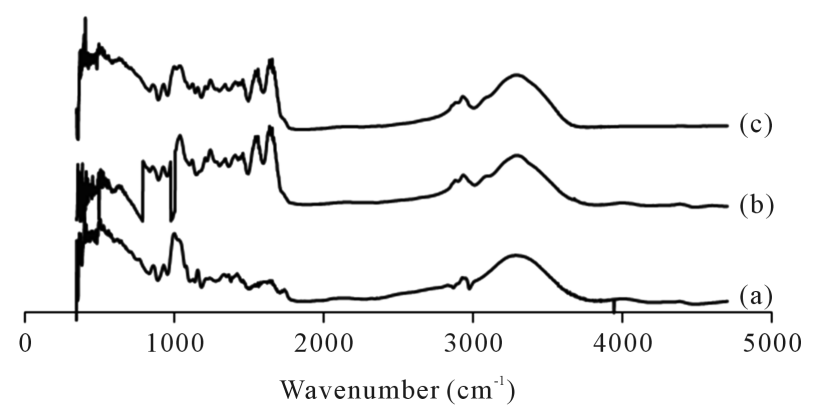

(B)

Figure 3. ATR-FTIR spectra of the ASS ((A)(a)), ApSS ((B) (a)) and $G((A)(c)$ and $(B)(c))$ plain films and ASS/G (50/50) ((A)(b)) ApSS/G (50/50) ((B)(b)) blend films.

\subsection{Thermal Capacity}

Differential scanning calorimetry (DSC) is a useful technique to reveal the variation in the thermal flow and to obtain the thermal critical points like melting point, enthalpy, specific heat or glass transition temperature. The variation of glass transition temperature $\left(T_{g}\right)$ is another effective indicator of the compatibility of polymers. DSC Thermograms of plain films of starch, gelatin, as well as starch/gelatin blend films were at different ratios of starch/gelatin are shown in Figure 4. It is worth noting, DSC in this study was taken place by performing one cycle of heating. However, the first heating scan cycle can be done in order to eliminate thermal history in the sample and also to eliminate moisture content, where starch and gelatin properties in solid state depend critically on the thermal history [17]. Generally, DSC thermograms for all of the plain and blend films, were typical of partially crystalline materials presenting a glass transition $\left(T_{g}\right)$ related to the amorphous part of the material, followed by endothermic peak, related to the melting $\left(T_{m}\right)$ of the crystals (see Table 2). It is worth noting, dehydrated gelatin properties in solid state depend critically on the thermal history [18]. DSC thermogram of ASS showed two weak endothermic peaks for glass transition and melting at $94^{\circ} \mathrm{C}$ and $274^{\circ} \mathrm{C}$, respectively. However, distinct two endothermic peaks at $110^{\circ} \mathrm{C}$ and $283^{\circ} \mathrm{C}$ in case of ApSS. The appearance of these endothermic peaks in thermograms of both types of starches can be due to occurrence of micro-crystallites that are held together by extended micellar network of associated polysaccharide molecules [18]. Since, the area under the endothermic peak expressed the heat of fusion of crystalline structure. The smaller area in case of ASS explains the lower crystallinity of ASS film. On the other side, two endothermic peaks represent glass transition of gelatin; the first is minor and appeared at $63^{\circ} \mathrm{C}$ while the second was more intense and appeared at $128^{\circ} \mathrm{C}$. These peaks were followed by melting peak appeared at $248^{\circ} \mathrm{C}$. These findings can be explained on the basis that the pure gelatin films are partially crystalline. Whereas, the glass transition endothermic peaks are associated to the sol-gel (helix-coil) transition. The block copolymer model for the amino acid content of the gelatin explains this occurrence [19]. The first glass transition temperature is associated with the glass transition of $\alpha$-amino acid blocks in the peptide chain, while the second glass transition temperature is represents the blocks of imino acids, proline, hydroxyproline with glycine. However, the following peak represents the fusion of the gelatin crystals, indicating the presence of a triple helical structure. 


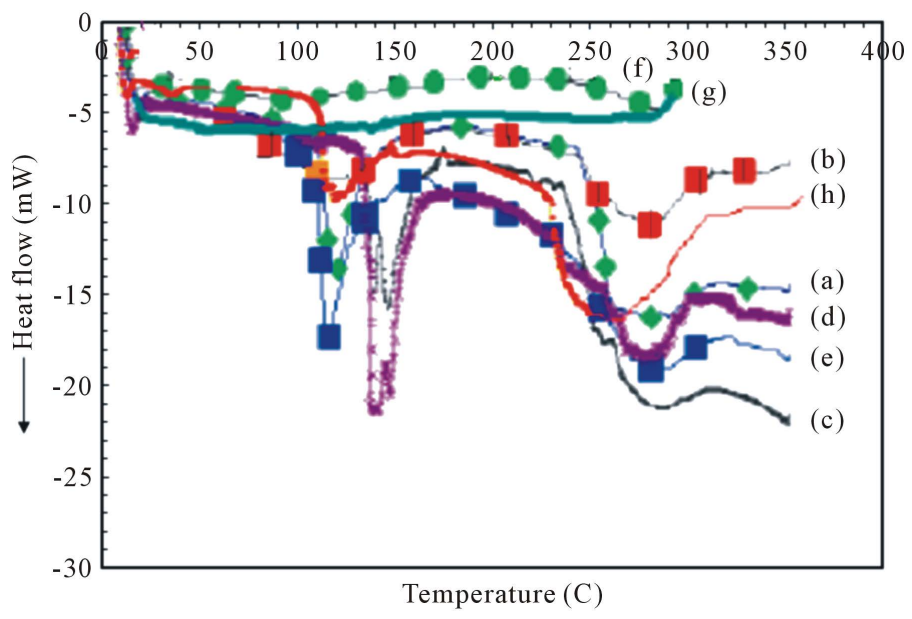

Figure 4. DSC thermograms of ApSS (a), ASS (f) and G (h) plain films and blend films; ApSS ApSS/G (90/10) (b); ApSS/G (80/20) (c); ApSS/G (70/30) (d); ApSS/G (60/40) (e) and ASS/G (50/50) (g).

Table 2. Crystallinity percentage calculated on basis of DSC and XRD analyses.

\begin{tabular}{cccc}
\hline Film type & $\Delta H_{c}(\mathrm{~J} / \mathrm{g})$ & Xc $(\%)^{\mathrm{a}}$ DSC & Xc $(\%)^{\mathrm{b}}$ XRD \\
\hline Gelatin & -21.89 & 33.63 & 36.48 \\
ASS & -41.70 & 32.38 & 35.23 \\
ApSS & -54.65 & 34.97 & 37.82 \\
$50 \%$ (ApSS) & -56.61 & 29.66 & 32.66 \\
$60 \%$ (ApSS) & -56.90 & 29.81 & 32.66 \\
$70 \%$ (ApSS) & -61.94 & 32.46 & 34.45 \\
$90 \%$ (ApSS) & -75.11 & 39.36 & 42.27 \\
$50 \%$ (ASS) & -59.95 & 27.46 & 30.31 \\
\hline
\end{tabular}

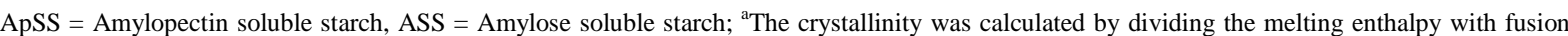
energy of $100 \%$ crystallinity for both of soluble starches and gelatin; ${ }^{\mathrm{b}}$ The crystallinity was calculated from the area under the amorphous peak and crystalline peaks of X-ray diffractogram.

On the other side, both of two types of soluble starch/gelatin blend films exhibited higher $T_{g}$ and $T_{m}$ values than basis of those for plain starch or gelatin films. Furthermore, they were increased with increasing starch content. This increase was more pronounced in case of amylopectin starch. When amylose soluble starch was blended with gelatin at ratio of 50/50, the melting peak was indistinct in the obtained thermograms. So, a single endothermic peak indicates high miscibility of amylase soluble starch with gelatin and homogeneity of the films. This is because the incorporation of starch into gelatin the matrix hinder its ability to form the triple helical structure (crystalline micro-domain), and consequently decreases the crystallinity of gelatin, resulting in a decrease in the degree of crystallinity and the heat of fusion of the blend films (see Table 2). On the other side, a sharp single endothermic glass transition peak in case of amylopectin soluble starch/gelatin films appeared in the range of $100^{\circ} \mathrm{C}-150^{\circ} \mathrm{C}$. Thus, appearing a more intense melting peak with slight shift to higher temperatures based on starch proportion. These findings confirm the partial miscibility of amylopectin soluble starch with gelatin with assumption of remaining the micro-crystallites of amylopectin soluble starch after its blending with gelatin [20].

\subsection{Crystallinity}

X-ray diffractograms (XRD) of plain soluble starch (ASS and ApSS), gelatin and blend films were shown in 
Figure 5. Amylose soluble starch films displayed a V-type XRD pattern (Figure 5(A)(a)) with characteristic peaks at $2 \theta=\sim 18^{\circ}$ and $20^{\circ}$ correspond to single helical conformation. However, the diffractogram obtained for amylopectin soluble starch (Figure 5(B)(a)) showed characteristic peaks located at $2 \theta=\sim 16^{\circ}$ and $19^{\circ}$ which confirm that this type of soluble starch is amorphous with occurrence of some granular structure (pseudo-crystalline). Crystallinity degree $\left(X_{c} \%\right)$ of soluble starch films estimated by measuring the area under the characteristic peaks of X-ray diffractogram where, it was found that the crystallinity index was $\sim 40 \%$ and $35 \%$ of amylopectin and amylose soluble starch film (Figure 6). These findings can be attributed to the integrity of some micro-crystalline domain in swollen granules that may be didn't completely disperse. The percentage of microcrystallites can be higher in ApSS comparing with that in ASS. Furthermore, negative charges of amylose single helices block their reorganization (retrogradation) with exerting a tension on neighboring crystallites of starch molecules and tended to distort those [21]. On the other side, gelatin film presented an X-ray diffraction pattern characteristic of a partially crystalline material, with a broad peak located at $2 \theta=\sim 20^{\circ}$, a sharp peak with low intensity located at $2 \theta=\sim 28^{\circ}$, and other in the region of $2 \theta=7^{\circ}$ to $8^{\circ}$ (Figure 5(f)). These characteristic peaks indicated the slight reconstitution of the collagen-like triple-helix structure.

Diffractograms of amylase soluble starch-gelatin films are shown in Figure 5(A)(b), Figure 5(A)(c), Figure 5(A)(d), and Figure 5(A)(e). A peak appeared more clearly defined at $10^{\circ}, 22^{\circ}$, and $30^{\circ}$. However, the crystalline peaks (at $18^{\circ}$ and $20^{\circ}$ ) of amylase soluble starch film were suppressed when the gelatin proportion in the blend film increased. The crystalline peaks of the blend films decreased because the added gelatin blocked the rearrangement of amylose strands. A broad amorphous peak with lower intensity observed in these patterns, demonstrating an interaction between these two components [22]. The decrease of crystallinity degree of the blend films was clearly noticed at polymer components ratio of 50/50 as shown in Figure 6. This decrease in crystallinity of the AAS/G blend films is mainly attributed to starch-gelatin interactions, where the polysaccharides could form networks with gelatin molecules between anionic domains of the polysaccharides and cationic domains of the gelatin. Therefore, the resultant ASS/G blend films were considerably more amorphous than

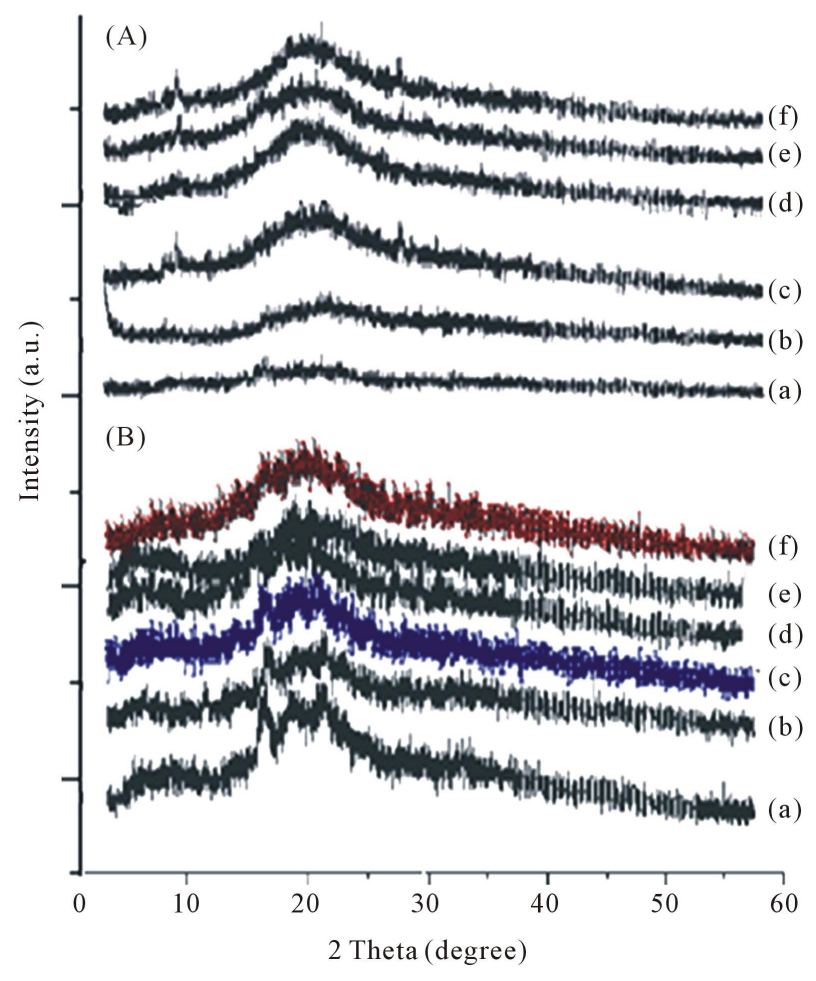

Figure 5. XRD diffractograms of the plain and ApSS/G (A) and ASS/G (B) blend films; plain ApSS and ASS films (Aa and $\mathrm{Ba})$; plain gelatin film ((A)(f) and $(\mathrm{B})(\mathrm{f}))$; ((A)(c)-(e) and (B)(c)-(e)) blends at starch/gelatin ratio of 90/10, 80/20, 70/30 and 50/50, respectively. 


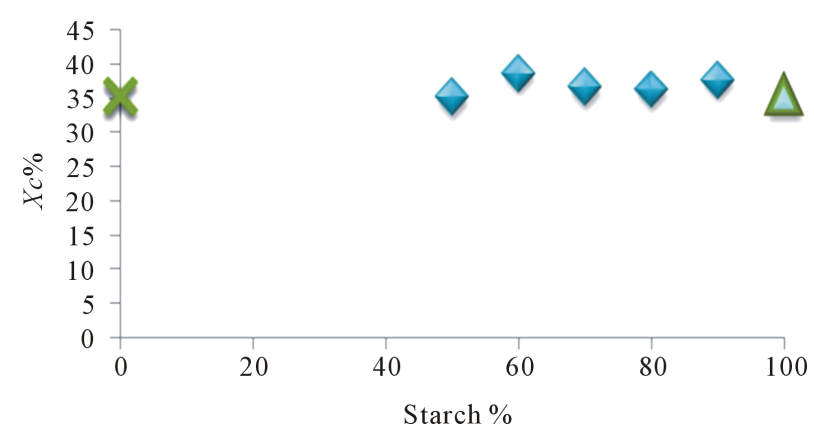

(A)

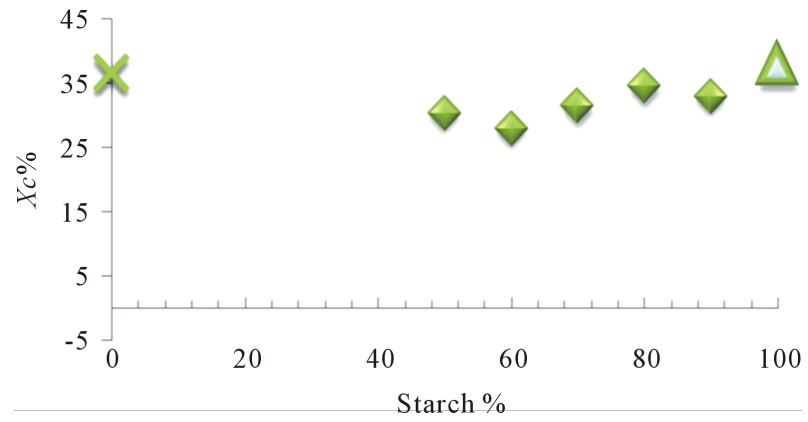

(B)

Figure 6. Effect of starch percentage on the degree of crystallinity (Xc\%) in ApSS/G (A) and AAS/G (B) blend films.

plain amylose soluble starch films. Similarly, blending of gelatin with amylopectin soluble starch/gelatin blend films, the intensity of the characteristic peaks of crystalline region of gelatin decreased with increasing the percentage of amylopectin soluble starch in their blends. These results can be explained on the basis that the incorporation of amylopectin can block the reconstitution of the gelatin helical structure. However, the amylopectin-gelatin interactions appeared to be less than those occurring between amylose and gelatin. Since the behavior of both of gelatin and amylopectin phases are to a higher extent independent. Therefore, the miscibility and compatibility of amylopectin soluble starch was found to be lower than those with its counterpart of amylose [23], Classically, a polymer-polymer interaction between polymer constituents of blend be at the expense of their crystallization process or is not in favor of crystalline domain formation for each of pure macromolecules. The crystallinity percentage in plain film of gelatin and soluble starch (ASS and ApSS) as well as starch/gelatin blend films were calculated based on DSC and XRD analyses and tabulated in Table 2.

Degree of crystallinity was calculated for pure gelatin film using DSC as previously reported based on the following equation:

$$
X_{c} \%=\Delta H_{m} / \Delta H 100 \% \text { re-natured }=\Delta H_{m} / \Delta H \text { collagen }
$$

where, $\Delta H$ for collagen $=62.05 \mathrm{~J} / \mathrm{g}$.

\subsection{Thermal Stability}

TGA thermograms of plain films of amylopectin and amylose soluble starch and gelatin as well as their blend films (in the temperature range $50^{\circ} \mathrm{C}$ to $800^{\circ} \mathrm{C}$ under nitrogen) are shown in Figure 7. In case of soluble starch films (ASS or ApSS), the initial weight loss at approximately $100^{\circ} \mathrm{C}$ was due to evaporation of water, while the second range $\left(200^{\circ} \mathrm{C}-450^{\circ} \mathrm{C}\right)$ corresponded to a complex process including depolymerization, dehydration of the saccharide rings. As shown in these thermograms, the starch films are stable up to $\sim 200^{\circ} \mathrm{C}$, with a maximum rate of decomposition occurring at $360^{\circ} \mathrm{C}$ or $410^{\circ} \mathrm{C}$ for amylopectin and amylose soluble starch films, respectively. The high amylopectin degradation rate larger than amylose starch may be related to the molecular size and branching fork structure of amylopectin, in addition to relatively higher crystallinity of amylose comparing with that of amylopectin starch as proven by findings of DSC and XRD analyses. On the other side, the TGA 


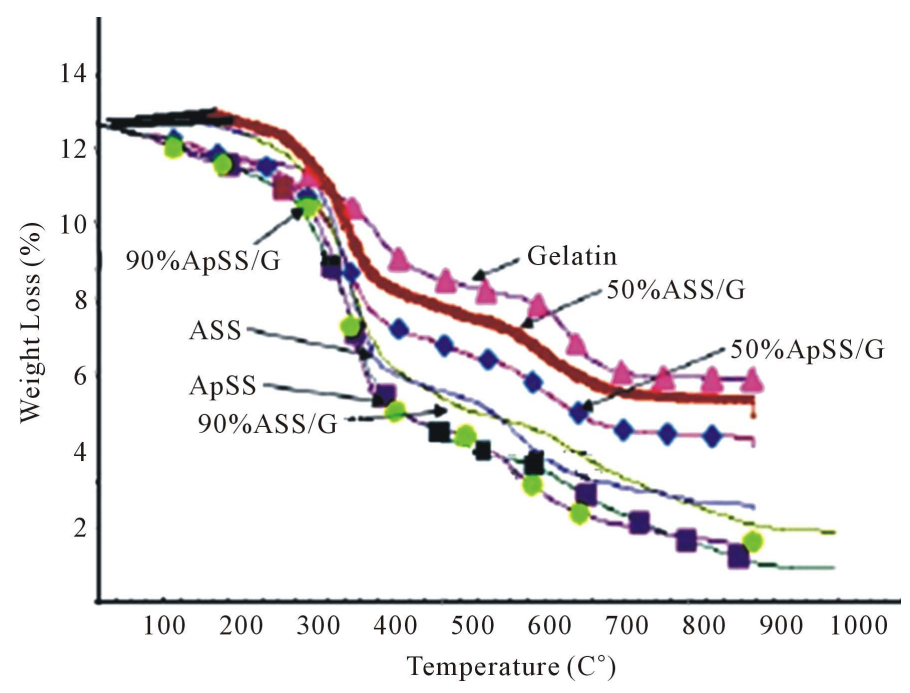

Figure 7. TGA thermograms of plain films of soluble starch and gelatin and starch/gelatin blend films.

thermogram of the gelatin films exhibit two regions of weight loss. The first weight loss at $30^{\circ} \mathrm{C}-150^{\circ} \mathrm{C}$ is attributed to mainly the loss of water, whereas the second weight loss takes place at $260^{\circ} \mathrm{C}-450^{\circ} \mathrm{C}$ which is associated with the protein chain breakage and peptide chain rupture. Further, the TGA thermograms of the blend films showed that the plain soluble starches films have earlier initial decomposition temperature than those for the plain gelatin and blend films. Thus, the initial thermal decomposition temperature shifted to a higher temperature with increasing the gelatin content in the blend films. The high thermal stability of these blends can be explained on the basis of increasing dissociation energy owing to numerous interactions occurring between the polymer blend constituents besides those acquired from gelatin addition [23].

\subsection{Mechanical Properties}

The results of the mechanical properties; tensile strength (TS) and elongation percentage (\% E) of the plain films of amylose soluble starch (ASS), amylopectin soluble starch (ApSS) and gelatin (as control) and soluble starches/gelatin blend films prepared in this study are shown in Figure 8(A), Figure 8(B) Since, Figure 8(A)(a)(b) showed that TS values were 1.79 MPa and 2.23 MPa for plain ASS film and ApSS film, respectively. However, TS for plain gelatin film of 7.5 MPa. TS value of the blend films was increased with increasing gelatin proportion in the blend films. Thus, this increase was more pronounced in case of ASS than those in case of ApSS. At equal proportion of starch and gelatin (1:1), TS value for ASS/gelatin blends was measured by 8.69 MPa while, it was 4.96 MPa for ApSS/gelatin ones. However, this difference became relatively negligible at higher starch proportion ( $\geq 90 \%$ ) so that TS values were comparable for both of two types of blend films. In order to get a better insight of the observed differences of tensile strength, it is noteworthy that the quaternary systems obtained by plasticization the soluble starch-gelatin systems with glycerol/water behave in a so complicated way where, interactions in starch/gelatin/water/polyol systems are polysaccharide/protein chains, polymer/water and polymer/polyol molecules, as well as between polyol/polyol or water/polyol molecules. Polysaccharides-protein interaction is made up from an average over the large number of different intermolecular forces arising between the various segments and side-chains on the two macromolecules [25]. Depending on the aqueous environmental conditions, the distribution of the different kinds of groups (charged, hydrophobic, hydrogen bonding, etc.) and the ratio of polymer constituents, the overall polysaccharide-protein interactions may be net attractive or net repulsive that govern miscibility of polymer constituents. The possibilities of gelatin or starch hydrogen-bonding within the blends are greatly enhanced by the introduction of comparatively small molecules such as water and polyols [26]. On the hand, temperature preparation and relative humidity greatly affect the tensile strength and percentage elongation of biopolymer films [27]. In the low-temperature film preparation, a two-stage process has been suggested: 1) formation of a gel and 2) its modification by contraction on drying. Conversely the high temperature method is known as the "one stage method". In this case, the molecules are considered to be in a 


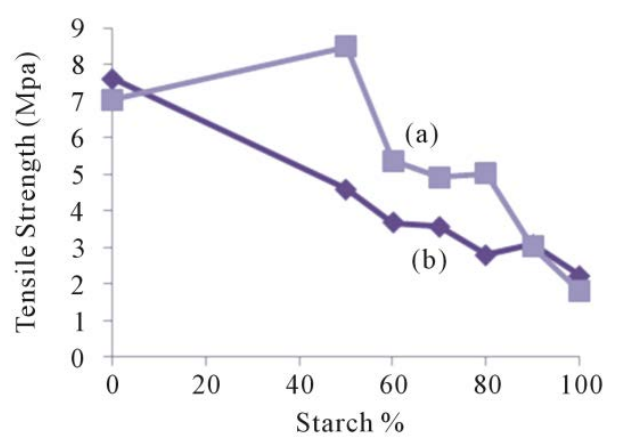

(A)

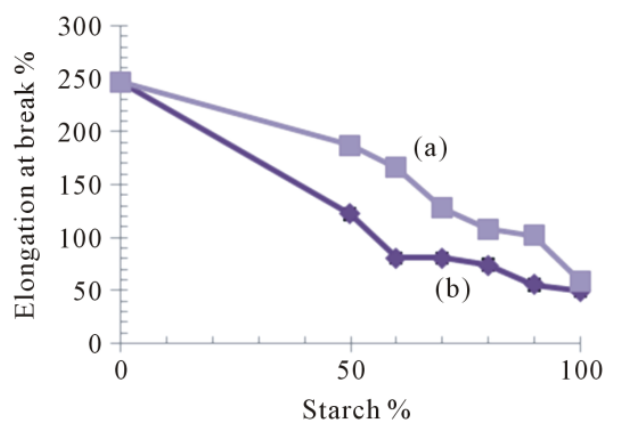

(B)

Figure 8. Effect of starch percentage on tensile strength (A), elongation-at-break (B) of ASS/G (a) ApSS/G (b) blend films.

closely packed condition at the time so that intermolecular bonding takes place. Consequently, any further growth in size of these bonded areas should be very limited because the molecules become entrapped in a randomly contracted state. Thus, thermal treatment or their previous thermal history affects significantly the mechanical properties of films via its impact on distribution and density of inter-molecular and intra-molecular interactions, which depend on the arrangements, and orientation of polymer chains in the network [28].

The elongation percentage ( $\% \mathrm{E}$ ), a measure of the flexibility, was recorded by $\sim 58.9 \%, \sim 49.2 \%$ and $\sim 247.3 \%$ for plain films of ASS, ApSS and gelatin, respectively (Figure 8(B)(a)(b)). Moreover, these results indicated that the different ratios of two biopolymers i.e. soluble starch (ASS or ApSS) and gelatin in blend films plasticized with a certain level of glycerol resulting in a significant effect on $\% \mathrm{E}$, where the film flexibility (\% E) increased when gelatin content was increased in all samples. However, this increase was also as TS more pronounced in cases of ASS blends. At soluble starch: gelatin ratio of 1:1, E\% was $\sim 179.6 \%$ and $~ 114.5 \%$ for ASS/G and ApSS/G blend films, respectively. The findings of this study suggested that despite of a reasonable plasticization effect of glycerol, gelatin seemed to act as a co-plasticizer which enhanced film flexibility and reduced brittleness. Since, findings of several researches reported that glycerol as a small size molecule that can penetrate between the polymer chains, and weaken the interaction between polysaccharides and proteins [29] [30]. However, others reported that the polysaccharide macromolecules with relatively long chains can crosslink with gelatin, leading to increased TS, and also possibly macromolecular relaxation leading to increase \% E [15] [31] [32]. Forasmuch an increase in relative crystallinity was detected with increase in starch proportion, particular in case of ApSS, it can be concluded that the strength and flexibility of the prepared soluble starch/ gelatin blend films could be modified by determining type of starch, the ratio of starch to gelatin and the preparation temperature.

\subsection{Barrier Properties}

Water vapor permeability (WVP) of plain films of ASS, ApSS and gelatin (as control) and series of soluble starch/gelatin blend films with various ratios of their components was examined at water vapor pressure gradient across the films of 0/60\% RH. The WVP values of plain and blend films were presented as a function of soluble 
starch/gelatin ratio in Figure 9(a), Figure 9(b). WVP was $\sim 23.13$ and $17.65 \mathrm{~g} \cdot \mathrm{mm} / \mathrm{m}^{2} \cdot \mathrm{day} \cdot \mathrm{kPa}$ for plain films of ASS and ApSS, respectively. However, WVP values were gradually decreased with increasing the percentage of gelatin in the blend films. Moreover, this decrease was more pronounced for ASS/G blend films than their corresponding ones of ApSS/G. Furthermore, the blend films of ASS/G, in general had a lower WVP values than those of ApSS/G counterparts. Where at starch/gelatin ratio of 90/10, WVP values recorded for blend films of ASS/G ApSS/G were $\sim 9.33$ and $\sim 15.45 \mathrm{~g} \cdot \mathrm{mm} / \mathrm{m}^{2} \cdot$ day $\cdot \mathrm{kPa}$, respectively. These values were found to be $\sim 6.46$ and $\sim 12.09 \mathrm{~g} \cdot \mathrm{mm} / \mathrm{m}^{2} \cdot$ day $\cdot \mathrm{kPa}$ for corresponding ones prepared with starch/gelatin ratio of 50/50.

These findings are is in contrast with those obtained by Jongjareonrak and others who reported that higher WVP was found with gelatin films containing greater protein content. Since forasmuch gelatin contained a wide range of hydrophilic amino acids, films with a higher amount of protein and thickness could absorb more water molecules from the environment [33]. Thus, the film with higher protein content was most likely to be hygroscopic, compared with that containing the lower protein content [34]. In other study, it has been reported that increasing protein content in the films that have varying the starch to gelatin ratio from 5:1 to 2:1 did not affect the WVP of both films with added sorbitol or glycerol [20]. However, Pranoto and his colleagues reported that the addition of gellan to gelatin films significantly reduced the WVP that may due to the ionic interaction between gelatin and gellan that formed a denser polymeric matrix, thus hindering water molecule transfer through the film. K-carrageenan was also found to form ionic complexes with gelatin but weaker than that of gellan/gelatin matrix [32].

WVP depends on many factors such as the ratio between crystalline and amorphous zone, polymeric chain mobility and specific interaction between the functional groups of the polymers and the gases in the amorphous zone [35]. Thus, WVP may be related to the difference in water molecule diffusion and hydrophilic/hydrophobic ratio [35] [36]. In the light of aforementioned, the findings obtained in this study can be explained on the basis that soluble starch-gelatin interactions was in favor of formation of compact dense network structure making the pathway of the permeate complex and decreasing its diffusion through the film matrices, and subsequently reduces the WVP. By other way, hydrophilic-hydrophilic interactions occurring between the biopolymer components of these blends can be transformed into more hydrophobic ones by thermal treatment leading to a decrease in WVP of the resultant films. On the other side, the difference in conformation of these two types of starch along with type and intensity of the potential starch-gelatin interactions are responsible for the noticeable differrences in the WVP of the obtained two blend films.

\subsection{Morphological and Topographic Properties}

Light microscopy was used to characterize the morphological and topographic of soluble starch/gelatin blend films (Figure 10(a)-(d)). The findings of this study showed that the starch and gelatin appeared clearly by two distinct colors in these films where, starch portion appeared to be stained by dark purple color (Iodine solution

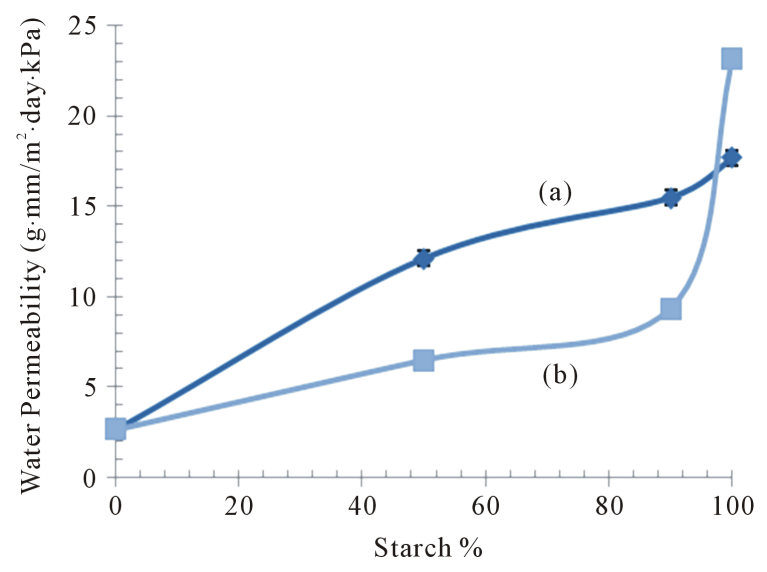

Figure 9. Effect of starch percentage on water vapor permeability (WVP) of AAS/G (a) and ApSS/G (b) blend films. 

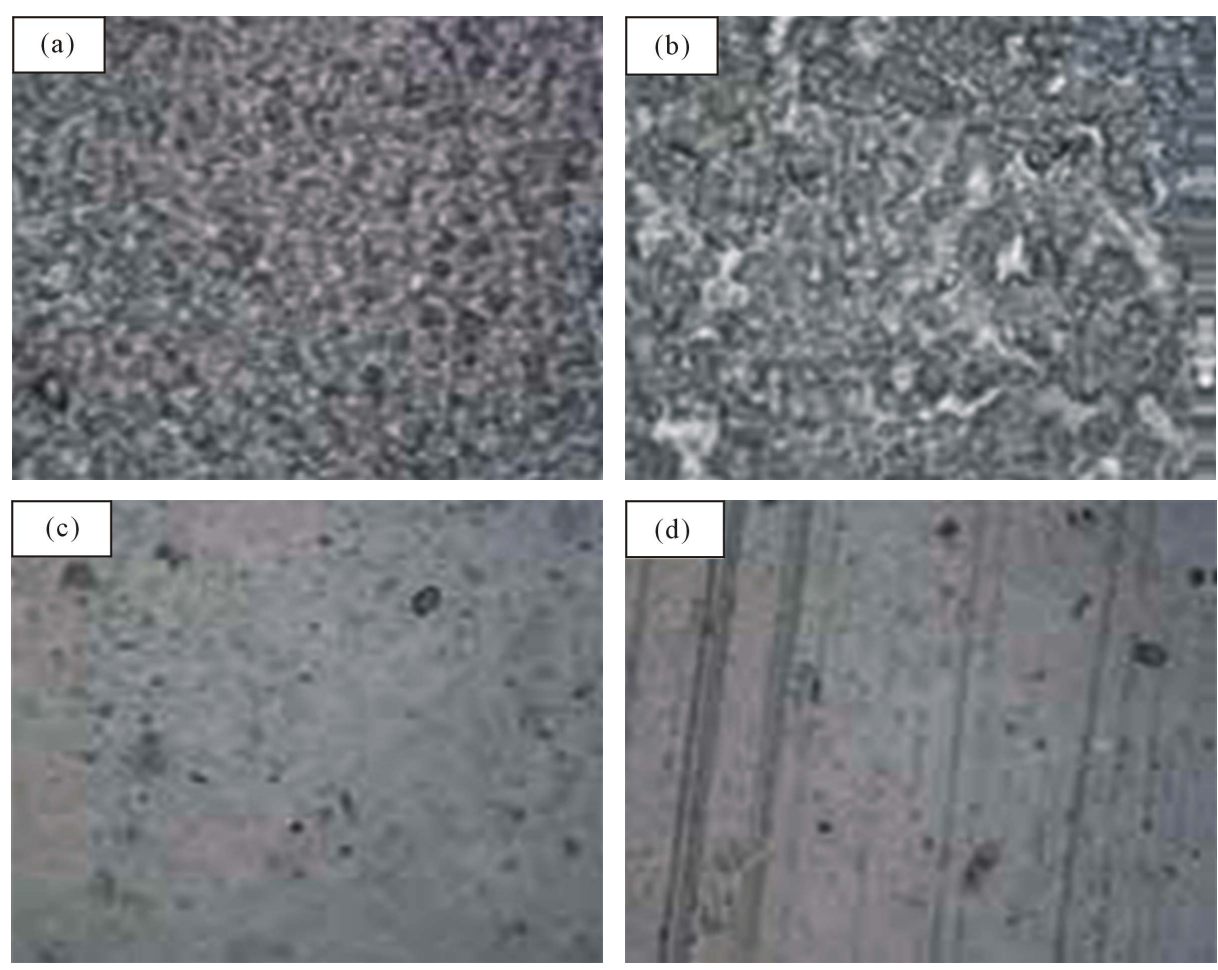

Figure 10. Light microscope photographs of soluble starch/gelatin blend films (ASS/G (50/50) (a) and (c) or ApSS/G (50/50) (b) and (d) at 500 and 1000×, respectively.

staining), while gelatin portion was unstained. By examination of these photographs, it is interesting to notice that such continuous amylose or amylopectin soluble starch networks (dark domain) are either entirely or partially surrounding by gelatin (light portion). Furthermore, no phase separation was noticed in both of blend films (50\% starch). These observations are in agreement with the results obtained from DSC thermograms of these blend films that revealed one endothermic peak. And thus with tensile curves that appeared to be synergetic in case of ASS/G blend films or even additive in case of ApSS/G blend films. Even though all aforementioned results prove that both of ASS and ApSS are miscible with gelatin, higher miscibility can be observed between ASS and gelatin that can be indicated from homogeneous distribution in the three dimensional structure of the blend films with surface was relatively smooth with less perturbations. This can be explained on the basis that both of amylopectin or amylose soluble starches are differently interacting with gelatin in their blends because of conformational changes in the macromolecular structure for ASS and ApSS. However, these results are not in agreement with those reported previously by Van Soet with others [37] who have confirmed that native starch and gelatin are considered immiscible polymer constituents that form separate phases. Further increase in proportion of gelatin component in the blend (10\% starch) led to increase homogeneity of the resultant films, whereas surface of these films appeared to be smooth. This can be attributed to that gelatin constituent forms the continuous phase (main domain), however the amylose or amylopectin soluble starch chains is the disperse phase that completely coved by the gelatin matrix.

\subsection{Micro-Structure Properties}

The surface and microstructure of soluble starch/gelatin films was examined using scanning electron microscopy. Based on the mechanical property results, a soluble starch blended film with $50 \%$ concentration of gelatin was chosen for surface and microstructure analysis. The scanning electron micrographs of these blend films are shown in Figure 11. The SEM micrograph of blend film (Figure 11(a)) showed a relatively smooth and continuous surface without pores and cracks, which confirmed a dense and homogeneous structure. Further, there is no phase separation as observed by light microscopy. However, surface roughness with perturbations disappeared in samples of blend films containing high proportion of gelatin (90\%) (Figure 11(b)). 

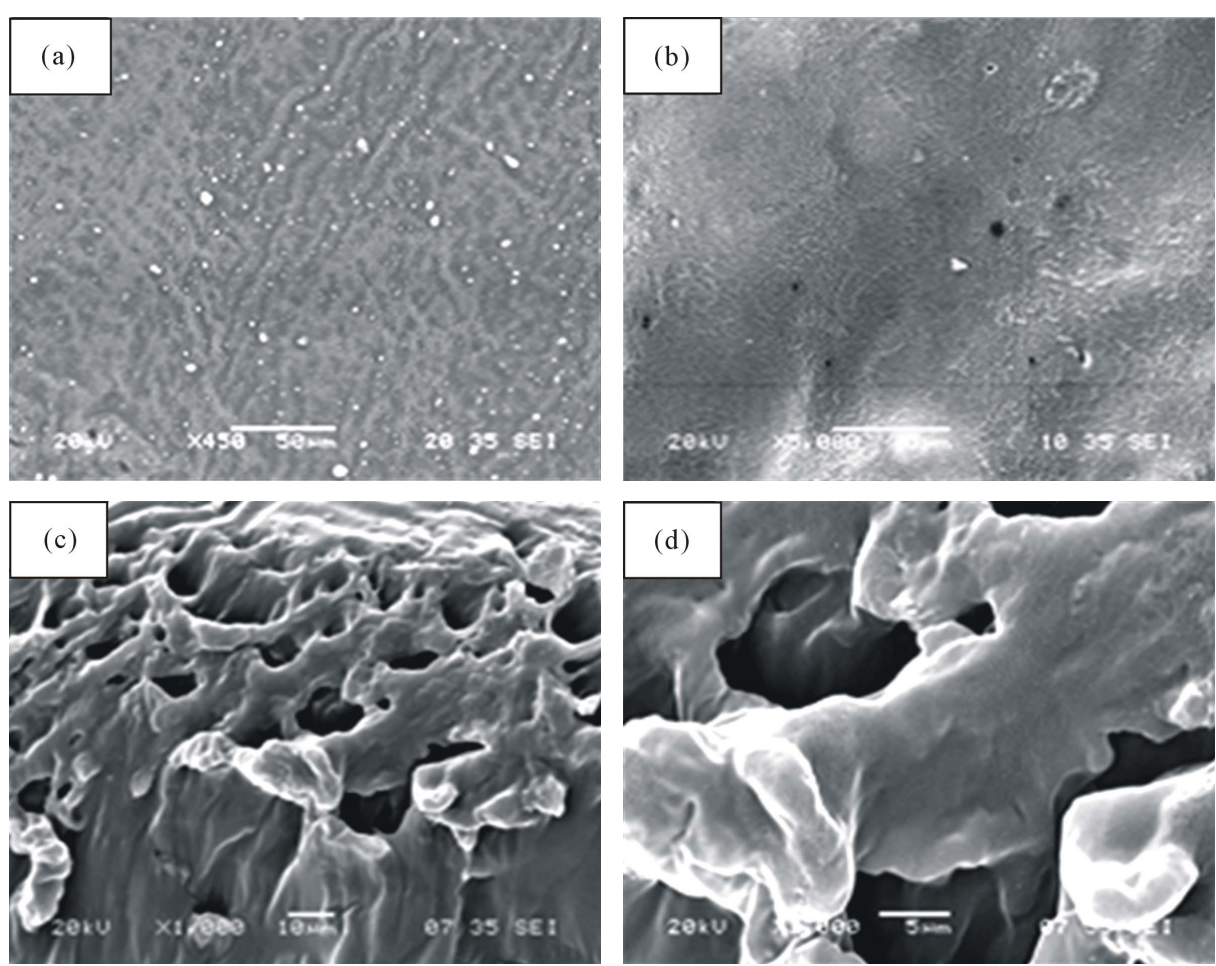

Figure 11. SEM micrographs of surface of blend films (ASS/G (90:10) (a) and ASS/G (50:50) (b)) and cross-section of ASS/G (50:50) at $1000 \times$ (c) or $3000 \times(d)$.

On the other side, microstructure micrographs of ASS/G blend film (1:1) exhibited that amylose chains appear in form of helices packed in regular arrays covered with a continuous phase of drawn gelatin. Conformation of dehydrated binary starch/gelatin systems is mainly amorphous network of helical amylose strands interacting with their counterparts of gelatin chains with formation of micro-crystallites that can act as mobile and nonpermanent cross-links. This conformation can be attributed to the compatibility of amylose and gelatin, constituents of blend films [13].

\section{Conclusion}

Blending of amylose and amylopectin soluble starches with gelatin to produce biodegradable films was a matter of this research. The physicochemical characterization, performed by Fourier transform infrared spectroscopy, differential scanning calorimetry, thermogravimetric analysis and scanning electron microscopy revealed presence of specific interactions among the functional groups of amylose soluble starch and gelatin resulting a good miscibility between these polymers which has lower than that occurred between amylopectin soluble starch and gelatin. The good miscibility in case of amylose soluble starches with gelatin acquired the resultant blend films good functional properties where, the tensile and water permeability resistance properties were enhanced. These improvements depended on starch/gelatin ratio.

\section{Acknowledgements}

This work was supported by a grant of Innovation Investment Initiative (III), under Research, Development and Innovation Programme (RDI), on behalf of the Scientific Research Ministry of Egypt.

\section{References}

[1] Lu, D.R., Xiao, C.M. and Xu, S.J. (2009) Starch-Based Completely Biodegradable Polymer Materials. eXPRESS Polymer Letters, 3, 366-375. http://dx.doi.org/10.3144/expresspolymlett.2009.46

[2] Ehring, R.J. (1992) Plastic Recycling. Hanser Publishers, Munich. http://dx.doi.org/10.1002/pi.4990300329 
[3] Brannock, G.R., Barlow, J.W. and Paul, D.R. (1991) Blends of Styrene/Maleic Anhydride Copolymers with Polymethacrylates. Journal of Polymer Science: Polymer Physics, 29, 413-429. http://dx.doi.org/10.1002/polb.1991.090290404

[4] Cowie, J.M.G., Elexpuru, E.M. and McEwen, I.J. (1991) Miscibility of Solution-Chlorinated Polyethylene with Poly ( $\alpha$-Methylstyrene-co-acrylonitrile). Journal Polymer Science: Polymer Physics, 29, 407-412. http://dx.doi.org/10.1002/polb.1991.090290403

[5] Kambour, R.P., Bendler, J.T. and Bopp, R.C. (1983) Phase Behavior of Polystyrene, Poly (2,6-Dimethyl-1,4-Phenylene Oxide), and Their Brominated Derivatives. Macromolecules, 16, 753-757. http://dx.doi.org/10.1021/ma00239a010

[6] Jagannath, J.H., Nanjappa, C., Das Gupta, D.K. and Bawa, A.S. (2003) Mechanical and Barrier Properties of Edible Starch-Protein Based Films. Journal of Applied Polymer Science, 88, 64-71. http://dx.doi.org/10.1002/app.11602

[7] Paul, D.R., Barlow, J.W., Keskkula, H. and Mark-Bikales, O.-M. (1988) Encyclopedia of Polymer Science and Engineering. 2nd Edition, John Wiley, New York, 399-405. http://dx.doi.org/10.1002/pol.1988.140260314

[8] Soliman, E.A., Tawfik, M.S., El-Sayed, H. and Moharram, Y.G. (2007) Preparation and Characterization of Soy Protein Based Edible/Biodegradable Films. American Journal of Food Technology, 2, 462-476. http://dx.doi.org/10.3923/ajft.2007.462.476

[9] Giita Silverajah, V.S., Ibrahim, N.A., Yunus, W.M.-Z.W., Abu Hassan, H. and Woe, C.B. (2012) A Comparative Study on the Mechanical, Thermal and Morphological Characterization of Poly (lactic acid)/Epoxidized Palm Oil Blend. International Journal of Molecular Sciences, 13, 5878-5898. http://dx.doi.org/10.3390/ijms13055878

[10] Sasaki, T. and Matsuki, J. (1998) Effect of Wheat Starch Structure on Swelling Power. Cereal Chemistry, 75, 525-529. http://dx.doi.org/10.1094/CCHEM.1998.75.4.525

[11] ASTM (2000) Standard Test Methods for Tensile Properties of Thin Plastic Sheeting, Method D882-00. American Society for Testing and Materials, Philadelphia. http://dx.doi.org/10.1520/D0882-00

[12] ASTM (2000) Standard Test Methods for Water Vapor Transmission of Materials, Method E 96-00. American Society for Testing and Materials, Philadelphia. http://dx.doi.org/10.1520/E0096-00

[13] Miya, M., Iwamoto, R. and Mima, S. (1984) FT-IR Study of Intermolecular Interactions in Polymer Blends. Journal of Polymer Science: Polymer Physics, 22, 1149-1151. http://dx.doi.org/10.1002/pol.1984.180220615

[14] Mousia, Z., Farhat, I.A., Pearson, M., Chesters, M.A. and Mitchell, J.R. (2001) FTIR Microspectroscopy Study of Composition Fluctuations in Extruded Amylopectin-Gelatin Blends. Biopolymers, 62, 208-218. http://dx.doi.org/10.1002/bip.1015

[15] Fonkwe, L.G., Narsimhan, G. and Cha, A.S. (2003) Characterization of Gelation Time and Texture of Gelatin and Gelatin-Polysaccharide Mixed Gels. Food Hydrocolloids, 17, 871-883. http://dx.doi.org/10.1016/S0268-005X(03)00108-5

[16] Brinke, G.T., Karasz, F.E. and MacKnight, W.J. (1983) Phase Behavior in Copolymer Blends: Poly(2,6-Dimethyl-1,4Phenylene Oxide) and Halogen-Substituted Styrene Copolymers. Macromolecules, 16, 1827-1832. http://dx.doi.org/10.1021/ma00246a006

[17] Zou, G.X., Jin, P.Q. and Xin, L.Z. (2008) Extruded Starch/PVA Composites: Water Resistance, Thermal Properties, and Morphology. Journal of Elastomers and Plastics, 40, 303-316. http://dx.doi.org/10.1177/0095244307085787

[18] Yannas, J.B. and Tobolsky, A.V. (1964) Viscoelastic Properties of Plasticized Gelatin Films. Journal of Physical Chemistry, 68, 3880-3882. http://dx.doi.org/10.1021/j100794a505

[19] Fraga, A.N. and Williams, R.J.J. (1985) Thermal Properties of Gelatin Films. Polymer, 26, 113-118. http://dx.doi.org/10.1016/0032-3861(85)90066-7

[20] Al-Hassan, A.A. and Norziah, M.H. (2012) Starch-Gelatin Edible Films: Water Vapor Permeability and Mechanical Properties as Affected by Plasticizers. Food Hydrocolloids, 26, 108-117. http://dx.doi.org/10.1016/j.foodhyd.2011.04.015

[21] French, D. (1984) Organization of Starch Granules. In: Whistler, R.L., BeMiller, J.N. and Paschall, E.F., Eds., Starch: Chemistry and Technology, 2nd Edition, Academic Press, New York, 183-195. http://dx.doi.org/10.1016/b978-0-12-746270-7.50005-7

[22] Hu, G., Chen, J. and Gao, J. (2009) Preparation and Characteristics of Oxidized Potato Starch Films. Carbohydrate Polymers, 76, 291-298.

[23] Arvanitoyannis, I., Psomiadou, E., Nakayama, A., Aiba, S. and Yamamoto, N. (1997) Edible Films Made from Gelatin, Soluble Starch and Polyols, Part 3. Food Chemistry, 60, 593-604. http://dx.doi.org/10.1016/S0308-8146(97)00038-1

[24] Arvanitoyannis, I., Nakayama, A. and Aiba, S. (1998) Edible Films Made from Hydroxypropyl Starch and Gelatin and Plasticized by Polyols and Water. Carbohydrate Polymers, 36, 105-119. 
http://dx.doi.org/10.1016/S0144-8617(98)00017-4

[25] Tolstoguzow, V.B. (1994) Some Physicochemical Aspects of Protein Processing in Foods. Multicomponent Gels. Food Hydrocolloids, 9, 317-332. http://dx.doi.org/10.1016/s0268-005x(09)80262-2

[26] Dickinson, E. (1998) Stability and Rheological Implications of Electrostatic Milk Protein-Polysaccharide Interaction. Trends in Food Science \& Technology, 9, 347-354. http://dx.doi.org/10.1016/s0924-2244(98)00057-0

[27] Bradbury, E. and Martin, C. (1952) The Effect of Temperature of Preparation on the Mechanical Properties and Structure of Gelatin Films. Proceedings of the Royal Society, Series A, 214, 183-192. http://dx.doi.org/10.1098/rspa.1952.0160

[28] Chambi, H. and Grosso, C. (2006) Edible Films Produced with Gelatin and Casein Cross-linked with Transglutaminase. Food Research International, 39, 458-466. http://dx.doi.org/10.1016/j.foodres.2005.09.009

[29] Mali, S., Sakanaka, L.S., Yamashita, F. and Grossmann, M.V.E. (2005) Water Sorption and Mechanical Properties of Cassava Starch Films and Their Relation to Plasticizing Effect. Carbohydrate Polymers, 60, 283-289. http://dx.doi.org/10.1016/j.carbpol.2005.01.003

[30] Su, J.F., Huang, Z., Yuan, X.Y., Wang, X.Y. and Li, M. (2010) Structure and Properties of Carboxymethyl Cellulose/Soy Protein Isolate Blend Edible Films Crosslinked by Maillard Reactions. Carbohydrate Polymers, 79, 145-153. http://dx.doi.org/10.1016/j.carbpol.2009.07.035

[31] Lee, K.Y., Shim, J. and Lee, H.G. (2004) Mechanical Properties of Gellan and Gelatin Composite Films. Carbohydrate Polymers, 56, 251-254. http://dx.doi.org/10.1016/j.carbpol.2003.04.001

[32] Pranoto, Y., Lee, C.M. and Park, H.J. (2007) Characterizations of Fish Gelatin Films Added with Gellan and K-Carrageenan. LWT-Food Science and Technology, 40, 766-774. http://dx.doi.org/10.1016/j.lwt.2006.04.005

[33] Jongjareonrak, A., Benjakul, S., Visessanguan, W. and Tanaka, M. (2006) Effects of Plasticizers on the Properties of Edible Films from Skin Gelatin of Big Eye Snapper and Brownstripe Red Snapper. European Food Research Technology, 222, 229-235. http://dx.doi.org/10.1007/s00217-005-0004-3

[34] McHugh, T.H., Avena-Bustillos, R. and Krochta, J.M. (1993) Hydrophilic Edible Films: Modified Procedure for Water Vapor Permeability and Explanation of Thickness Effects. Journal of Food Science, 58, 899-903. http://dx.doi.org/10.1111/j.1365-2621.1993.tb09387.x

[35] Garcia, M.A., Martino, M.N. and Zaritzky, N.E. (2000) Lipid Addition to Improve Barrier Properties of Edible StarchBased Films and Coatings. Journal of Food Science, 65, 941-944. http://dx.doi.org/10.1111/j.1365-2621.2000.tb09397.x

[36] Arvanitoyannis, I., Kalichevsky, M., Blanshard, J.M.V. and Psomiadou, E. (1994) Study of Diffusion and Permeation of Gases in Undrawn and Uniaxially Drawn Films Made from Potato and Rice Starch Conditioned at Different Relative Humidities. Carbohydrate Polymers, 24, 1-15. http://dx.doi.org/10.1016/0144-8617(94)90111-2

[37] Van Soest, J.J.G., Hulleman, S.H.D., De Wit, D. and Vliegenthart, J.F.G. (1996) Changes in the Mechanical Properties of Thermoplastic Potato Starch in Relation with Changes in $\beta$-Type Crystallinity. Carbohydrate Polymers, 29, $225-232$. http://dx.doi.org/10.1016/0144-8617(96)00011-2 